\title{
Research on Prevention and Control of Ebola
}

\author{
Dianjun Ju \\ School of Electric Power Engineering, North China Electric Power University, Baoding 071000, \\ China
}

jdj1058785624@163.com

Keywords: The delivery system; Time Satisfaction Degree; SEIR-D.

\begin{abstract}
In this paper, we will establish two realistic, sensible, and useful Ebola virus eradication models: a basic model. In the basic model, we consider several factors in the basic model: the quantity of the medicine needed, speed of manufacturing of the vaccine and drug, locations of delivery. First, the delivery system model is established to determine the location of the drug dispensing stations. Then, the basic Ebola virus propagation model is built by adopting SEIR-D construction with increased dead chamber D. Finally various factors are integrated into the basic model to obtain the integrated model. We conduct sensitivity analysis on the amount and time of drugs dispensing.
\end{abstract}

\section{Introduction}

The Ebola virus disease (EVD), known as Ebola hemorrhagic fever, outbreaks in West Africa. The three worst-affected countries are Guinea, Liberia and Sierra Leone, which are the background of our model.

The official website of World Health Organization (WHO) [1] has a special report for the Ebola virus. Most of the data we need can be found on this website. Meanwhile, we also analyze the geographic position in the Maps of World which offer detailed maps all over the world.

\section{Assumptions}

To simplify the problem, we make the following assumptions about the modeling process in this paper:

There are enough vehicles to deliver the vaccines and medicines.

Ensure the vaccines and medicines can be sent out when needed.

The Ebola virus only spread from person to person.

Ignore the virus is obtained from animals.

Taking no account of the influence of the climate, especially temperature.

Table1 Parameters of the Delivery System Model

\begin{tabular}{ccc}
\hline Parameter & Meaning & Units \\
\hline$S_{j}^{*}$ & $\begin{array}{c}\text { The selected conditions of candidate } \\
\text { locations }\end{array}$ & Unitless \\
& $\begin{array}{c}\text { The degree of drug dispensing station } S_{j} \\
\text { serving the affected area } D_{i}\end{array}$ & Unitless \\
& The longest waiting time of $D_{i}$ when Time & $\mathrm{h}$ \\
$T_{1 \text { long }}$ & Satisfaction Degree is 1 & $\mathrm{~h}$ \\
& The shortest waiting time of $D_{i}$ when Time \\
$T_{0 \text { short }}$ & Satisfaction Degree is 0 & \\
\hline
\end{tabular}




\section{Symbols, Terminology Definitions}

\subsection{Symbols and Definitions}

Table 2 Parameters of the Basic SEIR-D Ebola virus propagation model

\begin{tabular}{ccc}
\hline Parameter & Meaning & Units \\
\hline$N$ & The total population of a city & Unitless \\
$E$ & The number of susceptible & Unitless \\
$I$ & The number of preclinical people & Unitless \\
$R$ & The number of infectious people & Unitless \\
$D$ & The number of recovered people & Unitless \\
\hline
\end{tabular}

\section{Basic Model}

The main factor is the delivery system. So we firstly establish the delivery system. Then the basic Ebola virus propagation model is built by adopting SEIR-D construction. Finally various factors are integrated into the basic model to obtain the integrated model.

\subsection{The Delivery System Model}

Considering the economic condition, technical reserve, natural environment and other factors of West African, it is inappropriate to build pharmaceutical factories in West Africa. But in order to eliminate the Ebola virus, or at least its current strain, it necessary to set up delivery system [2] that guarantees optimal time.

The Time Satisfaction Function is defined as follow:

$$
\operatorname{TSD}\left(t_{i j}\right)=\left\{\begin{array}{cc}
1 & \left(t_{i j} \leq T_{1 \text { long }}\right) \\
\frac{T_{0 \text { short }}-t_{i j}}{T_{0 \text { short }}-T_{1 \text { long }}} & \left(T_{0 \text { short }} \leq t_{i j} \leq T_{1 \text { long }}\right) \\
0 & \left(t_{i j}>T_{0 \text { short }}\right)
\end{array}\right.
$$

The Modeling Process. Step 1: Given value of $S_{j}{ }^{*}$ and $F_{i j}$

$S_{j}^{*}=\left\{\begin{array}{l}1, \quad \text { the candidate position } S_{j} \text { is set } \\ 0, \quad \text { the candidate position } S_{j} \text { is not set }\end{array} ; \forall S_{j} \in S\right.$

$F_{i j}=\left\{\begin{array}{rr}1 & S_{j} \text { serves } D_{i} \\ 0 & S_{j} \text { do not serves } D_{i}\end{array}\right.$

Step 2: Objective function

We define that objective function is the total Time Satisfaction Degree that cover all affected areas.

Without considering the severity of the affected areas, the objective function is defined as follow:

$\max T S D=\sum_{i=1}^{m} \sum_{j=1}^{n} T S D\left(t_{i j}\right) F_{i j}$

Result \& Analysis

By solving equations, we can obtain the following results:

Conakry undertakes the delivery task to Gueckedou, Nzerkore and Lola.

Kissidougou undertakes the delivery task to Forecariah, Coyah and Dubreka.

Lola undertakes the delivery task to the rest of the cities in Guinea.

Bomi undertakes the delivery task to Bomi, Gbarpolu and Grand Cape Mount. 
Bong undertakes the delivery task to the rest of the cities in Liberia.

Kenema undertakes the delivery task to Kailahun, Kenema, Kono, Tonkolil, Bo, Bonthe, Pujehun and Western-rural.

Western Urban undertakes the delivery task to Bombali, Kambia, Port Loko, Koinadugu, Moyamba and Western Urban.

\subsection{The Basic SEIR-D Ebola virus propagation model}

According to the current knowledge about the ecology of Ebola disease, and considering the Ebola's high case fatality rate, it has large influence on transmission dynamics. So we increase the dead chamber D on the basis of the four chambers in SEIR. [3]

From the figure 2 , we can see that $N=S+E+I+R+D=$ const. So we can derive the follow formula:

$$
S=N-E-I-R-D
$$

The establishment of differential equations

$$
\begin{gathered}
\left\{\begin{array}{c}
\frac{d E}{d t}=e(N-E-I-R-D)-i E \\
\frac{d I}{d t}=i E-r I-d I \\
\frac{d R}{d t}=r I \\
\frac{d D}{d t}=d I
\end{array}\right. \\
e(N-E-I-R-D)=e S
\end{gathered}
$$

That is the number of susceptible transforming into the EVD carriers.

\section{Result \& Analysis}

We assume that the drugs are delivered from the15th day after a certain time nodes. We assume that the dosage can supply total three thousand people for the treatment every day.

We draw to reflect the quantity of dose every day in the three countries. Origin of coordinates represent the first day after dosing.

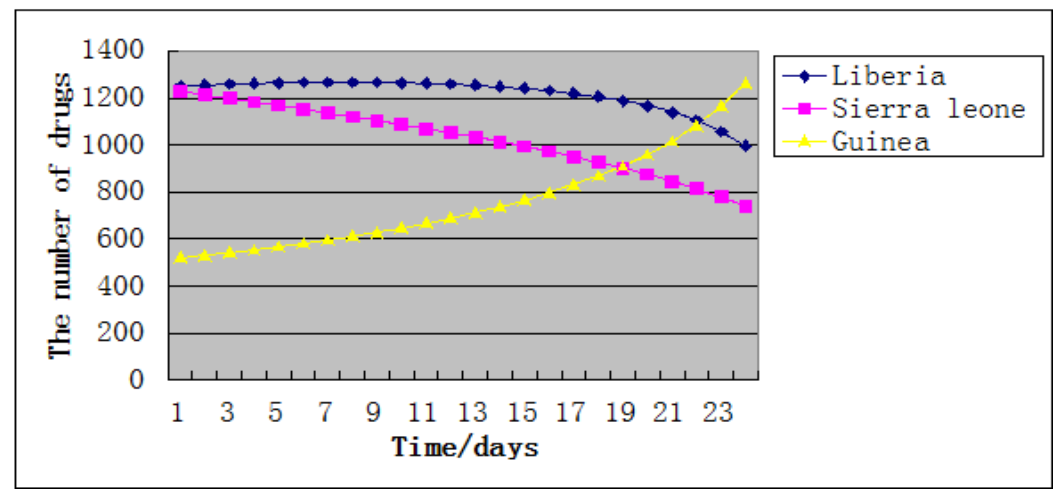

Figure 1 The change trend of the quantity of dose

\subsection{Analysis}

The quantity of supplying drugs is the dynamic feedback model, so the quantity of supplying dru gs for each country is dynamically changing.

\subsection{Sensitivity analysis}

We assume that the drugs are delivered from the15th day and the dosage can supply total 3000 people for the treatment every day. Then change the time into the tenth day.

We dispense the drugs and vaccines to supply the treatment of the 3000 patients from the 15 th day, and the pressure of outbreak will be relieved to some extent. As we dispense the drugs every day, the 
number of people who are infected the Ebola decreases gradually. The epidemic situation has been controlled fundamentally after about 40 days.

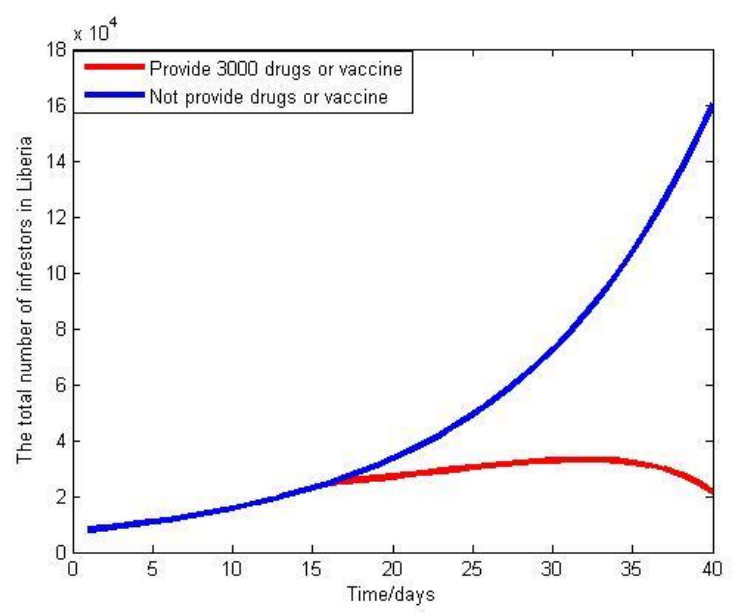

Figure 2 Infectors delivered from the 15th day

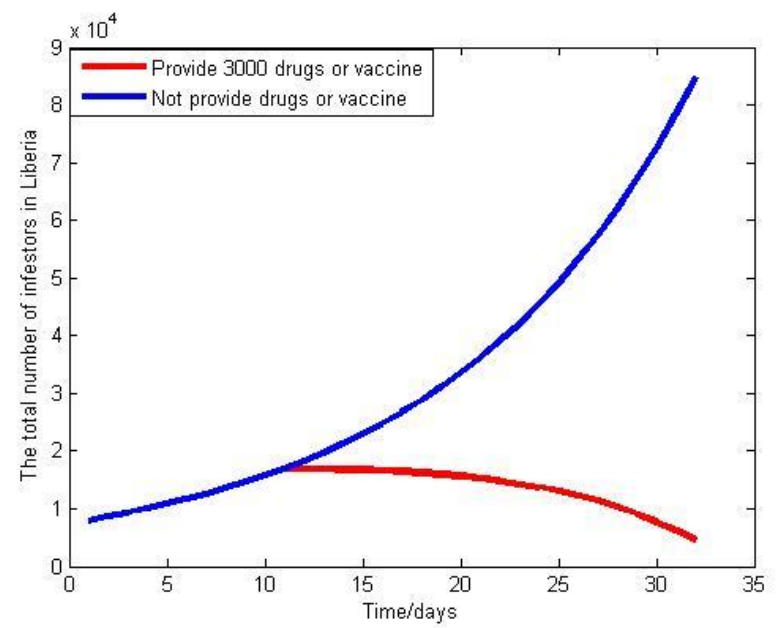

Figure 3 Infectors delivered from the tenth day

\section{References}

[1]http://www.who.int/en/

[2] Manhua Yao.Study on the Site Selection of Coastal Search and Rescue Base[D].Dalian: Daliann Maritime University,2011:30-32.

[3] W O Kermack, A G McKendrick. Contributions to the mathematical theory of epidemics. Proc. Roy. Soc. 1927, (All5): 700-721. 